mass concentration without the whole Universe collapsing under its own gravity down into the singularity, like a gigantic house of cards?

A further problem concerns the precise details of the recycling mechanism, whereby the heavy elements (ashes of the stars) are flushed down towards the singularity and the replacement light elements, such as hydrogen, which are used to build up new generations of stars, drift up towards us.

Needless to say there are also a number of philosophical problems. Naked singularities are usually regarded as a serious disease in physics, something to be avoided at all costs. Most relativists support Roger Penrose's idea of a cosmic censor who always clothes singularities inside black holes, where they cannot influence the outside world. In the Ellis model the singularity-by definition a lawless and unpredictable creature-is contrived to drive, ultimately, all the organised activity in the Universe.

There is also the curious problem of why, if the Universe is infinitely old and life is concentrated in our particular corner of the cosmos, it is not inhabited by technological communities of unlimited age. Is technology recycled too? In a cryptic footnote to his paper Ellis comments that it may be desirable to find a compromise model Universe, incorporating features both of the standard expanding model and his own new ideas.

In spite of all the difficulties, this remarkable new slant on the cosmic structure and organisation serves at least as a reminder that the conceptual basis of modern cosmology can be widened. Perhaps the Copernican revolution is over?

\section{Superfluid ${ }^{3} \mathrm{He}$ at Manchester}

\section{from P. V. E. Mc Clintock}

Now that the superfluid phases of liquid ${ }^{3} \mathrm{He}$ (discovered at Cornell University in 1972) are no longer quite such a novelty, it seems timely to pause, take stock of what has been achieved and consider how best to tackle the substantial problems still remaining. It had always been clear that a number of the early experiments paid insufficient attention to charac-

P. V. E. McClintock is a member of the Department of Physics at the University of Lancaster. terising the textures exhibited by the (highly anisotropic) superfluids; and it was entirely fitting, therefore, that $\overline{\mathbf{a}}$ recent meeting on superfluid ${ }^{3} \mathrm{He}$ at Manchester* should have been notable for people's concern about how to predict and control the textures taken up by the liquid under different sets of experimental conditions.

D. Bailin (University of Sussex) described the ways in which topology could contribute to an understanding of the textures and transitions between them. Although he sought to emphasise that because one does not feed very much information into a topological analysis one cannot reasonably expect to get much out again in the way of quantitative conclusions, it became clear in the course of his talk that a good deal of insight may nonetheless be gained by this approach.

One method of controlling the texture experimentally lies in careful choice of the shapes of the surfaces with which the liquid comes in contact: it was reported that the Sussex NMR experiments were being conducted on liquid held in fine tubes $1 \mu \mathrm{m}$ in diameter; whereas, at Orsay, the liquid was contained within a stack of $0.4 \mathrm{~mm}$ Mylar plates. Other factors affecting the texture were the applied magnetic field, which was easy to control, and heat flow, which was not. The effect of a flow of heat was expected to be quite complicated and it could even give rise to 'dynamical textures', that is, textures which vary systematically with time, according to J. R. Hook (Manchester University). It seemed very likely that the long lived periodic motions observed earlier by J. C. Wheatley and his coworkers at La Jolla were a consequence of this effect.

J. D. Reppy (Cornell University) described recent high precision experimental results obtained at Cornell using what amounts to a modified Andronikoshvili apparatus: an inverted torsion pendulum whose hollow interior could be filled with liquid ${ }^{3} \mathrm{He}$. By measuring changes in the characteristic frequency and damping coefficient attributable to the ${ }^{3} \mathrm{He}$, values could be deduced for the average viscosity and superfluid density, and interesting comparisons could then be made with viscosities measured by a completely different method employing a vibrating wire.

Summing up and pointing towards possible future developments, A. J. Leggett (Sussex University) discussed some of the puzzles still being posed by superfluid ${ }^{3} \mathrm{He}$. Coping effectively with textures presented severe theoretical difficulties, and he enthusiastically

*Held on 28 April. endorsed an earlier remark by Hall to the effect that hoping to predict textures from the equations of superfluid hydrodynamics was almost as ambitious as expecting to be able to predict the weather by solving the Navier-Stokes equations! An experimental approach was best in the first instance, and he went on to discuss the techniques, such as ultrasound, already available as well as an interesting, and as yet unproven, idea involving possible reflection of quasiparticles when they enter regions where the energy gap, relative to their direction of motion, is decreasing. Other important but unresolved puzzles included: the absence of the electric field effect (electric fields had been expected to orientate the $l$ vector, but experimentally they apparently failed to do so); the magnitude of the A-phase liquid's intrinsic angular momentum (a bucket of initially normal liquid ${ }^{3} \mathrm{He}$ should start to rotate when cooled into the superfluid A-phase, but how fast?); anomalous differences between static and NMR measurements of the Bphase magnetic susceptibility; and the range of validity of the (so far, remarkably successful) 'giant diatomic molecule' picture of the superfluids. The latter model has, of course, recently received something of a boost through Leggett's own successful use of it for predicting, correctly, that the A-phase would be weakly ferromagnetic.

\section{More puzzles about the early Solar System}

\section{from $M$. Edmunds}

THE determination of the relative abundances of isotopes in meteorites continues to produce unexpected results. A particularly copious and fruitful source of this early Solar System material has been the meteorite from Allende in Mexico. The relatively high abundance of ${ }^{26} \mathrm{Mg}$, the decay product of ${ }^{26} \mathrm{Al}$, in some aluminium-rich minerals in this meteorite has already prompted suggestions that heavy elements freshly made in a supernova explosion were incorporated into solid material during the formation of the Solar System (see News and Views 267, 393; 1977). Anomalies in the isotopic

M. Edmunds is in the Department of Applied Mathematics and Astronomy at University College, Cardiff. 